${ }^{1}$ Department of Internal Medicine, Hypertension and Vascular Diseases, Warsaw Medical University, Warsaw, Poland

2Department of Invasive Cardiology, Central Clinical Hospital of the Ministry of Interior and Administration, Warsaw, Poland

${ }^{3}$ Department of Invasive Cardiology, Centre of Postgraduate Medical Education, Warsaw, Poland

\title{
A bumpy road to the diagnosis of metastatic lung cancer
}

\author{
Corresponding author: \\ Jacek Bil, Department of Invasive \\ Cardiology, Centre of Postgraduate \\ Medical Education, Woloska Street 137, \\ 02-507 Warsaw, Poland, \\ e-mail: jacek.bil@cmkp.edu.pl
}

\begin{abstract}
:
Cardiac tumors pose the uncommon group of disorders and most of them are metastases. They are generally localized in the left heart and may present with non-specific symptoms. Diagnosis may be often difficult due to the presence other heart diseases. The gold standard in diagnosing is non-invasive imaging. We present a case of a 77-year-old male admitted to our hospital due to flu symptoms, headaches and loss of weight in the last 2-3 weeks. The diagnostic process including computed tomography (CT) and magnetic resonance imaging revealed pathological contrast enhancement in the left parietal lobe, whereas subsequent transthoracic echocardiography disclosed a pathological oval mass $2.5 \times 3.5 \mathrm{~cm}$ in the left heart suspected of a metastasis. Results of echocardiography turned the diagnostic process around and extended to bronchoscopy and abdominal CT. Finally, central left lung tumor with metastases to lungs, pleura, lymphoid nodes of the right hilar, left ventricle, left suprarenal gland and central nervous system was diagnosed.

Key words: cardiac tumor, non-invasive imaging, transthoracic echocardiography, metastasis, cardiac metastasis
\end{abstract}

Med Res J 2020; 5 (3): 211-214

\section{Introduction}

Transthoracic echocardiography (TTE) is widely known as a non-invasive method to characterize the structure of the heart, its dimensions, blood circulation and other physical aspects. It may also describe cardiac hypertrophy, which is classified as concentric or eccentric change based on relative heart wall thickness. Cardiac hypertrophy may have a different background, e.g. hypertension, valve stenosis, endocrine disorders or genetic aspects. Also, cardiac tumors are rare disorders which result in heart wall hypertrophy. It is less likely to find the primary tumor, because most of them are metastases. The incidence of metastases to the heart is underestimated and may be up to $25 \%$ according to some post-mortem studies [1]. Lung cancer, that has the highest prevalence in cardiac metastases, is the most commonly diagnosed cancer in the world and associated with the largest number of cancer-related deaths [2, 3]. Computed tomography (CT) is a basic diagnostic modality of lung cancer, and the timely diagnosis of cardiac metastases may improve prognosis by faster diagnosis of the primary tumor and help to prevent acute complications like cardiac tamponade or pulmonary edema.
We present a case of a male in whom the TTE gave an opportunity to make a diagnosis and vitally change the treatment.

\section{Case presentation}

We present a case of a 77-year-old male with a history of hypertension, hyperthyroidism (treated with radioiodine therapy) and with right mediastinum enlargement, which was under observation since 2001 (most likely goitre). In 2011 the patient was offered surgical removal of suspected tumor but did not agree to proceed. The patient was admitted to our hospital due to fever present in the last 2-3 weeks (up to $39^{\circ} \mathrm{C}$ ), dry cough, lack of appetite, increasing tiredness and weight loss. Before admission, the patient was treated with oral antibiotics (amoxicillin + clavulanic acid followed by ciprofloxacin) with no improvement and with persistent fever. At admission, the patient was stable, with no fever. During physical examination, no abnormalities were found except for paleness. The abnormal laboratory test findings included: WBC $-13.11 \times 10^{9} / \mathrm{L}$, serum fasting glucose $-168 \mathrm{mg} / \mathrm{dL}$, C-reactive protein 
- 154.1mg/L, and serum creatinine - 1.60mg/dL. Ambulatory chest radiogram apart from the lesion in the right mediastinum did not reveal any abnormalities. In chest radiogram at admission, additional opacities in the right hilar were noted and described as suspected of being segmental atelectasis due to compression, as well as atelectasis in the left costodiaphragmatic recess, were mentioned. Ultrasonography of the abdomen was normal. Blood cultures were twice negative. The patient was treated with ceftriaxone. No fever was observed, cough decreased, the decision about discharge was made. At the day of the discharge, a patient's family mentioned severe headaches the patient was suffering from for several weeks before admission. According to them headaches required large doses of nonsteroidal anti-inflammatory drugs the patient was taking on his own, also during current hospitalization (the probable reason for the lack of fever). Neurologist consulting the patient recommended head MRI, which was made and revealed at least 7 areas of pathological contrast enhancement, largest measuring $12 \mathrm{~mm}$ localized in left parietal lobe and no other meaningful abnormalities (Fig. 1A). Taking all under consideration diagnosis of multiple abscesses was made. As a part of the further diagnostic process in order to find abscesses origin TTE was performed. It revealed slight left atrium enlargement, cardiac effusion with a maximum thickness of $12 \mathrm{~mm}$, hypertrophy of the posterior and lateral left ventricular (LV) wall with an oval mass $-3.5 \times 2.5 \mathrm{~cm}$. Cardiomyopathy to be differentiated with inflammatory infiltration and the cardiac tumor was suggested (Fig. 2). Above-mentioned LV wall showed contractility abnormalities, but no signs of valve disease or infective endocarditis were found. At that point, taking under consideration that the most common tumors of the heart are metastases, a neoplastic process with multiple metastases started to be suspected. Chest and abdominal

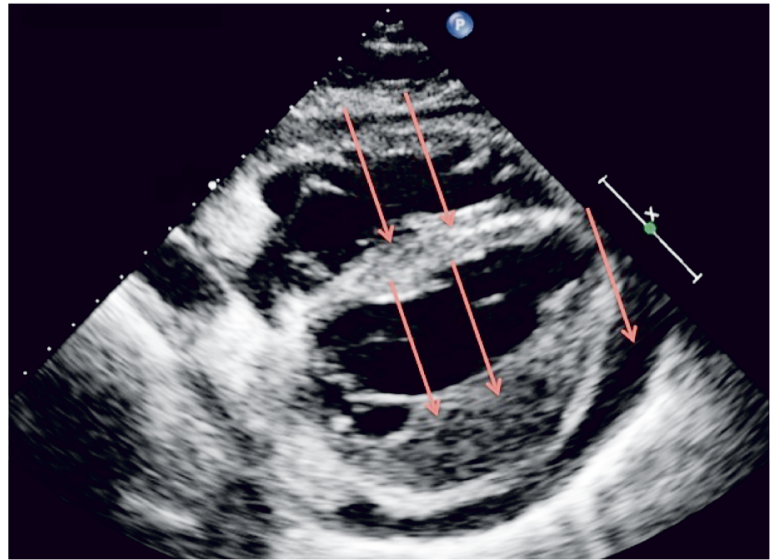

Figure 2. Transthoracic echocardiography showing the infiltration of the intraventricular septum, the oval mass, and the effusion in the pericardium

CT and bronchoscopy were performed. Bronchoscopy revealed in the area of carina of trachea a pathological vascular network, and a concentric edema of the initial segments of the left secondary bronchi with stenosis of the left inferior lobar bronchus origin. Chest and abdominal CT showed a pathological mass with signs of necrosis and maximum size of $6 \mathrm{~cm}$ in the right hilar (Fig. 1B), with amputation of one of the right pulmonary artery branches, with right inferior lobar bronchus blockage, and with enlarged lymph nodes in the right hilar. A mass was interfusing with a right main bronchus wall and segmentally with a descending aortic wall. Atelectasis secondary to bronchi compression in the right inferior pulmonary lobe were also noted. Several lesions suspected of metastases in the right lung were also present. Lesions suspected of metastases were also present in the LV wall - localizing from a basal anterior segment, through antero- and inferolateral segments,

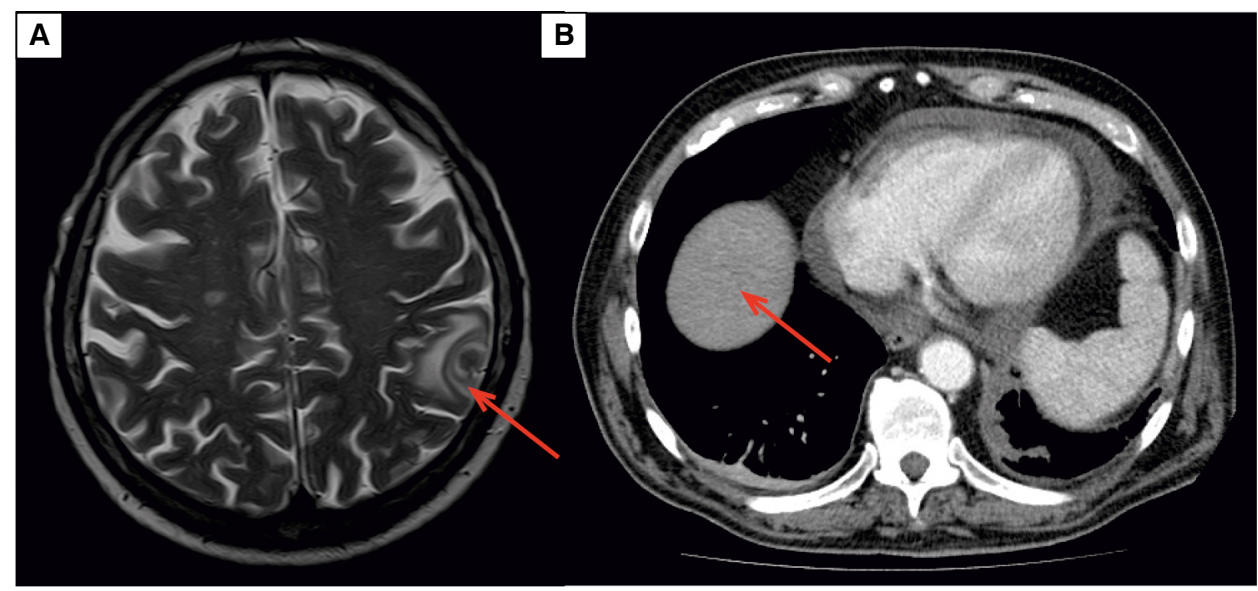

Figure 1. A. Magnetic resonance imaging showing the metastatic lesion in the left parietal lobe; B. Computed tomography showing the large primary tumor in the right lung 
infiltrating epicardium, with pericardial effusion. Also, similar lesions were present in the intraventricular wall. Additionally, metastases in the left suprarenal gland and pulmonary pleura were present. The lesion in the upper right mediastinum was described as polycystic, with a size of $8.5 \mathrm{~cm}$ - most likely to be a goiter. The central left lung tumor with metastases to lungs, pleura, lymph nodes of the right hilar, left suprarenal gland, LV and intraventricular wall were diagnosed. Taking all into account, lesions in the central nervous system were also diagnosed as metastases, and the patient was referred for further treatment to the oncology department.

\section{Discussion}

Heart muscle hypertrophy may have different etiologies: physiological in the athlete's heart, or rather pathological, i.e. in hypertension, valvular heart disease (stenosis), hypertrophic cardiomyopathy or a neoplastic process.

Transthoracic echocardiography is one of the main imaging modalities used in differentiating the cause of hypertrophy. In our case hypertrophy of the posterior and lateral LV walls with an oval mass was diagnosed. It suggested cardiomyopathy to be differentiated with inflammatory infiltration and cardiac tumor. Although taking into account full clinical picture metastatic lesions seemed the most probable and the patient was further diagnosed in that direction.

Cardiac tumors belong to a group of rare clinical disorders with a frequency, depending on the population study taken into account, differing between $0.0017 \%$ and $0.33 \%$. Only $5 \%$ are classified as primary tumors [4]. Most of them are metastases. However, their clinicopathological state has not been explicated yet [5]. Nevertheless, few potential primary types can be distinguished: myxomas (being the most popular - 72.6\%), followed by fibromas (6.9\%) and sarcomas (6.4\%) [6].

Cardiac metastases are most common in patients in the sixth and seventh decade of life, without sex preferences. Generally, every malignant tumor can metastasize to the heart, but the most common are carcinomas of the lung, breast, esophagus, lymphoma, leukemia and melanoma [7]. Lung cancers make up over $38 \%$ of all metastases to the heart (adenocarcinoma $-14.6 \%$; poorly differentiated $-12.4 \%$; squamous cell $-11.8 \%$ ) [3]. However, clinical picture and cardiac symptoms depend mainly on the size and localization of the tumor than its histological type $[1,7]$.

The diagnosis is extremely difficult due to its non-specific symptoms, that can be wrongly attributed to other cardiac diseases. Moreover, cardiac metastases remain often asymptomatic for a long time, therefore heart involvement is often not noticeable till death
[7]. While symptoms are present, they may manifest as heart rhythm disturbances, conduction disorders, angina pectoris, dyspnea or cough [1]. Metastases to pericardium are preferable from lung and breast cancers, and they may lead to a pericardial effusion [7]. It is one of the most feared complications since may lead to death. It can be mild and give slightly symptoms like in our case; however, it may also increase rapidly and require pericardiocentesis [3].

Heart metastases are often not only the the first, but also the only change that indicates the presence of a malignant tumor [3]. At the time of diagnosis, metastases are often not limited to the heart [8]. The situation was similar in our case, at the moment of the diagnosis, the patient had not only metastases to the heart but also to other organs, i.e. the suprarenal gland and the right lung. In addition, the tumor infiltrated vital organs - descending aorta and left main bronchus.

There are no guidelines for the treatment of cardiac metastases, mainly due to low incidence and lack of evidence $[8,9]$. Treatment is generally focused on the underlying tumor [10]. Treatment of lung cancer may be divided into surgical and non-surgical i.e. chemotherapy, radiotherapy, immunotherapy. The choice of method of the treatment depends on the histological diagnosis and stage and the methods may be combined to increase effectiveness [11]. Cardiac treatment is mainly limited to palliative measures [7]. Surgery may be considered in symptomatic patients to alleviate the symptoms and/or in selected cases to remove the tumor [8]. Resection may be performed among patients without metastases in other organs when the tumor can be removed completely [9]. Among inoperable and asymptomatic patients, nonsurgical treatment should be initiated [8]. Since chemotherapy and tumor resection may alleviate symptoms and prolong survival, our patient was referred to the oncology department [6].

\section{Conclusions}

Cardiac metastases are rare disorders and occur mostly in older patients. They may be the first and the only disorder indicating an active tumor process. Predominantly, metastases to the heart origin from lung cancer. In our case, taking all into account, the primary left lung tumor with metastases, including those in the heart wall was diagnosed. Our case suggests that despite unspecific symptoms, one of the simplest imaging modalities like TTE may still turn the diagnostic process around and help to give an accurate diagnosis.

\section{Acknowledgements: Authors declare no conflict of interest.}




\section{References}

1. Hudzik B, Miszalski-Jamka K, Glowacki J, et al. Malignant tumors o the heart. Cancer Epidemiol. 2015; 39(5): 665-672, doi: 10.1016/j. canep.2015.07.007, indexed in Pubmed: 26239627.

2. Hirsch FR, Scagliotti GV, Mulshine JL, et al. Lung cancer: current therapies and new targeted treatments. Lancet. 2017; 389(10066): 299-311, doi: 10.1016/S0140-6736(16)30958-8, indexed in Pubmed: 27574741

3. Bussani R, De-Giorgio F, Abbate A, et al. Cardiac metastases. J Clin Pathol. 2007; 60(1): 27-34, doi: 10.1136/jcp.2005.035105, indexed in Pubmed: 17098886.

4. Petris AO, Alexandrescu DM. Costache, II: Cardiac tumors. Rev Med Chir Soc Med Nat lasi. 2014; 118(2): 289-292.

5. Akishima S, Umezu Y. [Three cases of cardiac tumor; an approach to excise a large tumor in the left atrium]. Kyobu Geka. 2014; 67(12): 1075-1078, indexed in Pubmed: 25391470.
6. Ragland MM, Tak T. The role of echocardiography in diagnosing space-occupying lesions of the heart. Clin Med Res. 2006; 4(1): 22-32, doi: 10.3121/cmr.4.1.22, indexed in Pubmed: 16595790.

7. Reynen K, Köckeritz U, Strasser RH. Metastases to the heart. Ann Oncol. 2004; 15(3): 375-381, doi: 10.1093/annonc/mdh086, indexed in Pubmed: 14998838

8. Mkalaluh S, Szczechowicz M, Torabi S, et al. Surgical Treatment of Cardiac Metastases: Analysis of a 13-Year Single-Center Experience. Thorac Cardiovasc Surg. 2019; 67(8): 659-664, doi: 10.1055/s-00381667319, indexed in Pubmed: 30092599.

9. Hoffmeier A, Sindermann JR, Scheld HH, et al. Cardiac tumors--diagnosis and surgical treatment. Dtsch Arztebl Int. 2014; 111(12): 205-211, doi: 10.3238/arztebl.2014.0205, indexed in Pubmed: 24717305.

10. Palaskas N, Thompson K, Gladish G, et al. Evaluation and Management of Cardiac Tumors. Curr Treat Options Cardiovasc Med. 2018; 20(4): 29 doi: 10.1007/s11936-018-0625-z, indexed in Pubmed: 29556752.

11. Jassem J. Cancer of the lung, pleura and mediastinum. Oncology in Clinical Practice. 2019; 15(1) 